\title{
Sheep in Wolf's Clothing: The Role of Artifacts in Interpretive Schema Change
}

\author{
Stefan W. Konlechner ${ }^{1}$ Barbara Müller ${ }^{1}$ - Wolfgang H. Güttel ${ }^{1}$ • \\ Irina Koprax ${ }^{1}$ Karin Link ${ }^{1}$
}

Received: 8 May 2015 / Accepted: 28 June 2015 / Published online: 28 June 2016 (C) The Author(s) 2016. This article is available at SpringerLink with Open Access.

\begin{abstract}
In this paper, we investigate the role of artifacts in a failed project that aimed at implementing a new culture of dealing with errors in a hospital by transferring safety standards from the aviation industry. We apply the interpretative method of objective hermeneutics to elucidate the role of artifacts as linking pins between diverging interpretive schemata and collective action during attempts to modify organizational routines. In particular, we show how the implementation of artifacts may serve as a means to satisfy a new espoused schema, while at the same time they are created and interpreted in ways that strengthen the old enacted schema. Although on the surface everyone would appreciate changes in treatment routines that help to avoid errors, the guiding norms of individual vigilance and self-centeredness, a culture that emphasizes hierarchy as a core value as well as the lack of sanctions for enacting the old schema led to a situation where the new espoused schema was never enacted. Instead, artifacts were used to stabilize a divergence between espoused and enacted schemata. Failure remained a cultural taboo.
\end{abstract}

S. W. Konlechner

stefan.konlechner@jku.at

B. Müller

barbara.mueller@jku.at

W. H. Güttel

wolfgang.guettel@jku.at

I. Koprax

irina.koprax@jku.at

K. Link

karin.link@jku.at

1 Institute of Human Resource and Change Management, Johannes Kepler University Linz, Altenberger Str. 69, 4040 Linz, Austria 
Keywords Artifacts · Interpretive Schema - Organizational Routines · Organizational Change · Objective Hermeneutics

\section{JEL Classification L2 · M1}

\section{Introduction}

In recent decades the health care sector has been increasingly adopting procedures and standards from the aviation industry with the aim of increasing patient safety (Tamuz and Thomas 2006, 919). Implementing new safety standards in the health care industry requires managing organizational change processes in health care organizations. Managing organizational change, however, is far from easy, especially if it involves "second-order change" (Bartunek 1984), which focuses on altering interpretive schemata that capture shared assumptions, values and social expectations (Balogun and Johnson 2005; Elsbach et al. 2005). Changing long-established patterns of collective action often requires such a cognitive reorientation of the organization (Gioia et al. 1994) by espousing new or different interpretive schemata (Isabella 1990; Labianca et al. 2000). Extant research that addresses the question as to how interpretive schemata and collective action are interrelated (e. g. Feldman 2003; Howard-Grenville 2005; Tucker and Edmondson 2003) indicates that there is a recursive relationship between both, meaning that they mutually influence each other (Rerup and Feldman 2011, 602). Yet how espoused schemata are enacted and which mechanisms contribute to this enactment has remained under-investigated.

In this paper, we draw on empirical data from a large change project in the health care industry to extend current research on the interconnectedness of interpretive schemata and collective action. In particular, we draw on the concept of organizational routines as "repetitive, recognizable patterns of interdependent actions, carried out by multiple actors" (Feldman and Pentland 2003, 95) and interpretive schemata as "ways of thinking" to investigate a change project that aimed at enhancing hospital safety standards. The project focused on transferring best practices and procedures, such as the implementation of an error-reporting system and checklists, as well as human factor training, from the aviation industry to the health care context as part of a large risk-management project. One of the core aims of the project was to develop a new set of assumptions and values regarding how organization members deal with errors and near-misses by implementing new artifacts that should facilitate the modification of particular organizational routines, such as treatment routines or surgery. Originally, our intention was to analyze how the use of artifacts makes this transfer of good practices work. Our analysis, however, revealed that the change project had failed to fulfill its original intention. We then went on to investigate the reasons and mechanisms behind this failure.

In contrast to extant research that addresses the interrelationship of interpretive schemata and organizational routines and mainly emphasizes how espoused schemata become more and more enacted over time (e. g. Rerup and Feldman 2011), our results elucidate mechanisms that stabilize a divergence between espoused and enacted schemata. We propose that artifacts serve as linking pins that 
may be (ab)used to disconnect interpretive schemata and collective action. In our particular case, the artifacts serve as a means to satisfy the new espoused schema, while at the same time they are created and interpreted in ways that strengthen the old enacted schema. We show that changing organizational routines by creating new artifacts cannot be successful if these artifacts are - like sheep in wolf's clothing used in a way that still satisfies prevalent social norms, which are deeply embedded in the collective understanding of organization members and, thus, are further enacted in shared interpretive schemata. Although the new artifacts are purported to facilitate change, they remain harmless and enable the further enactment of established actions. Thus, contrary to our expectations, we found that how artifacts are used may serve as a way to decouple schemata from actions. This decoupling keeps the organization in a state of equilibrium that impedes learning.

\section{Conceptual Background}

This study focuses on the analysis of the recursive relationship between interpretive schemata and organizational routines as patterns of collective action. Although their close interaction is undisputed, interpretive schemata and organizational routines have developed as two independent research streams. Just recently the interdependence of both concepts has been made subject of discussion and stimulated attempts toward an integration of both concepts (e. g. Rerup and Feldman 2011). From a social practice perspective (e. g. Feldman 2004; Howard-Grenville 2005), we understand interpretive schemata as ways of thinking and organizational routines as courses of collective action. Artifacts are "objectified summaries of routines" (Feldman and Pentland 2003, 109), and therefore serve the purpose of connecting interpretive schemata and collective action. This section further elaborates on the applied concepts, demonstrates their overlap, and explores their interconnectedness.

Interpretive Schemata - Espousing Versus Enacting An interpretive schema can be defined as "a set of shared assumptions, values, and frames of reference that give meaning to everyday activities and guide how organization members think and act" (Rerup and Feldman 2011, 578). As generalized cognitive frameworks (Labianca et al. 2000, 236), interpretive schemata contain "fundamental (though often implicit) assumptions about why events happen as they do and how people are to act in different situations" (Bartunek 1984, 355). They incorporate social expectations (Howard-Grenville 2005) and communicate values and norms (Gioia et al. 1994). Thus, they provide meaning to organization members and justify behavior in organizations.

Research shows that although changing established organizational interpretive schemata is difficult (Nystrom and Starbuck 1984; Reger and Palmer 1996), they can be changed (Bartunek, Lacey, and Wood 1992; Labianca et al. 2000). Such change, however, requires the articulation of a new vision that contains new social expectations and values. Rerup and Feldman $(2011,578)$ refer to such new or different schemata as espoused interpretive schemata. Espoused interpretive schemata can be compared to the notion of an "in progress frame of reference" (Isabella 1990, 17), 
insofar as they are designed to replace existing schemata and to enable managers as well as organization members to develop new approaches in dealing with problems and challenges (Rerup and Feldman 2011, 578; Labianca et al. 2000, 238). Because "( $p$ )eople in different groups (Donnellon et al. 1986, Meyerson and Martin 1987), functional areas (Strasser and Bateman 1983), or hierarchical or status levels (Smith 1982) often see what is apparently the same event very differently" (Labianca et al. 2000, 237), espousing a new schema serves the purpose of aligning different ways of thinking and creating a common mindset within an organization. In contrast to espoused interpretive schemata, which contain an articulated vision, an enacted schema captures the expectations and values that actually guide collective behavior. It becomes observable as intention is transformed into a pattern of realized cognition and action (Rerup and Feldman 2011, 579). Organizational change is successful when the espoused schema and the enacted schema converge and new values start to determine individual and collective action (Balogun and Johnson 2005).

Organizational Routines and the Role of Artifacts The concept of organizational routines has emerged as one of the most powerful concepts for explaining collective action in organizations (Seidl and Becker 2006). Understanding organizational routines is thus a major prerequisite for understanding organizations (Becker 2004; Parmigiani and Howard-Grenville 2011; Salvato and Rerup 2011). Organizational routines are repetitive and recognizable collective phenomena that involve multiple actors who are connected by interaction (Feldman and Pentland 2003; Rerup and Feldman 2011). There are different opinions regarding the extent to which individuals are assumed to be able to actively influence organizational routines. Becker (2004, 648), for instance, distinguishes the view of routines as being "mindless" from the view of routines as being mindful and effortful accomplishments. While the view of routines as being mindless seems to be rather an implicit assumption of early research, current research increasingly conceptualizes routines not only as mindful, but even as multi-minded (e. g. Feldman and Pentland 2003; Rerup and Feldman 2011). The idea of multi-mindedness involves the assumption that individual actors, who act on the basis of their individual interpretations, influence the collective understanding of a routine with their actions (Feldman and Pentland 2003; Feldman 2004; Howard-Grenville 2005). As a consequence, changing mindsets and interpretive schemata on the individual, group, and organizational levels may be the first step in active attempts to change collective action.

Artifacts represent the visible symbols of organizational routines, e. g. formal rules, standard-operating procedures, or codified knowledge, such as "how-to" manuals (D‘Adderio 2008, 2011; Feldman and Pentland 2003). They provide individual actors with points of reference (Rafaeli and Vilnai-Yavetz 2004, 671). Accordingly, attempts to influence the collective performance of organizational routines usually involve designing and implementing new artifacts (Güttel et al. 2012; Leidner 1993). However, although artifacts "are often used to try to ensure the reproduction of particular patterns of action" Pentland and Feldman (2008, 241), they rely on the interpretation of individual actors to unfold their impact. Pentland and Feldman (2008, 242) use the following example to illustrate this phenomenon: "Consider the difference between a sign that says 'Employees only - Do not enter' and a 
locked door. (...) the sign relies much more on the interpretation than does the locked door. Some of the artifacts that surround organizational routines are more like locked doors in that they truly constrain action. But the vast majority, such as rules, forms, diagrams and procedures, are more like the sign in that their meaning is open to a variety of interpretations". Although it is tempting to assume that changing interpretive schemata and collective action patterns is solely a question of pushing the right buttons by implementing new artifacts, designing new routines or altering established ones is a complex and error-prone process, which requires understanding of the organization members' ways of thinking and collective courses of action. How artifacts actually connect such "ways of thinking" and "collective actions" is still a question that deserves more attention.

\section{Methods}

We applied a case study design (Eisenhardt and Graebner 2007; Yin 2009) for investigating the dynamics of interpretive schema change. Qualitative approaches facilitate grasping the complexity of organizational learning processes. They are even more advantageous when knowledge about organizational phenomena is unsatisfactory. In such cases, qualitative research is helpful to provide rich descriptions and build or refine theory (Eisenhardt 1989; Siggelkow 2007).

Research Setting Legal regulations in Austria require hospitals to regularly report quality measurement and performance indicators to the government. Consequently, hospitals are bound to perform quality management on the level of processes, structures and outcomes. Empirical findings indicate that transferring techniques and procedures to minimize incidents, such as checklists, team training, etc., from the aviation to the health care industry has positive effects on quality (Gawande 2011). As a consequence, this transfer of safety standards from aviation to health care is also gaining increasing attention in research (e. g. Tamuz and Thomas 2006; Lewis et al. 2011).

Originally, we started our investigation with the aim of uncovering how the introduction of artifacts facilitates this transfer of good practices in an Austrian hospital. The hospital has over 350 beds. In 2010, more than 700 employees were engaged in patient care. In order to increase the quality of patient care and to apply for external quality certification, the hospital management initiated a "clinical risk management" project in 2010. A consulting firm, which we have given the fictitious name "Riscure", was awarded the project because of its experience in improving hospital safety standards.

The aim of the consulting project was to identify all types of errors in order to develop measures for preventing them in the future. The project aimed at implementing a safety culture and changing treatment routines by increasing standardization through the implementation of new artifacts. The risk management project consisted of various subprojects. In order to improve patient safety, Riscure consultants introduced (1) an IT-based error reporting system, which we will call "FARES". Another measure was (2) the introduction of checklists for treating patients and for conduct- 
ing treatment and surgery. In addition (3) human factor training was implemented, with the intention of raising awareness of the importance of cross-functional and cross-hierarchical communication.

Data Collection To analyze the change project, we conducted 14 in-depth interviews with different representatives of the diverse hierarchical levels of the three core sub-groups in the hospital (medical staff, nursing staff, and administrative staff) and one interview with the CEO of the consulting firm Riscure who managed the implementation process between 2010 and 2013. All interviews were conducted in the mother tongue of interview partners and researchers.

The theoretical sampling of interview partners allowed us to interview all the important actors involved during the consulting process. The medical director, the head of nursing, and the quality manager are the key members of the "collective leadership board", which is the main body responsible for strategic decisions. On the employee level, we interviewed people in different hierarchical positions, including assistant medical directors, assistant doctors, nursing staff, and employees responsible for patient transport from different departments. The interviews lasted between 45 and 90 minutes. We recorded and transcribed all interviews verbatim for further analysis. Additionally, documents and observations helped us to triangulate our data. During the data analysis stage, we recognized the Janus-faced character of artifacts in our case. We therefore started to particularly analyze those mechanisms that foil change attempts.

Data Analysis If we had analyzed our data with a focus on the content level only, we probably would have identified a "good practice example" of the implementation of practices to enhance safety standards in hospitals. Actually this was the reason why we chose the case. The CEO of the consultancy company as well as important decision-makers in the hospital named this project as one of "their most successful projects", to put it in the words of the consultancy company head.

However, although the project initially seemed to indeed be a case of the successful implementation of practices applied in aviation to enhance safety standards in a hospital, one of the researchers quickly discovered some contradictions when starting to analyze the interview data. In particular, there seemed to be a significant gap between the manifest responses of our interview partners ("how they say they perceive failure") and the latent meaning behind what was said ("how they actually perceive failure and deal with it").

To analyze the reasons for these contradictions, this researcher decided to analyze the data following the approach of objective hermeneutics (Oevermann et al. 1979), which facilitates analyzing the deep structure of a text and the exposure of latentmeaning structures. Objective hermeneutics does not focus on reconstructing individual interpretations, but rather on uncovering "latent structures of meaning that underlie social practices and subjective meanings" Lueger et al. (2005, 1147). Although objective hermeneutics has a long tradition in German sociological research (cf. Flick 2002; Hildebrand 2004; Hitzler 2005), and is best suited to "analyze real social phenomena, to discover structures of meaning, and to reconstruct decisions 
and decision patterns by individuals, groups or organizations" (Wagner et al. 2010, 5), organization and management research drawing on this method is still scarce.

A notable example of the application of objective hermeneutics in organization research can be found in the work of Lueger et al. (2005, 1148), who used objective hermeneutics to analyze influence strategies and build on the assumption that "the meaning of an act only unfolds in relation to the social context it is embedded in". Objective hermeneutics, hence, facilitates deeper analyses than traditional content analysis. It is particularly useful to analyze how a system works and therefore well suited to answer "why" and "how" questions in organization research. In our analysis we followed the approach outlined by Lueger et al. (2005).

In our case, two teams of three researchers each analyzed different key paragraphs that the one researcher who knew about the interviews and the project in more detail at that stage had selected in a first step. Accordingly, each team analyzed key paragraphs they did not have any further context about - one of the most important preconditions for this kind of analysis. The teams began analyzing one single unit of meaning, the smallest part of a sentence that makes sense on its own, after the other without reference to subsequent text passages. Every unit of meaning was analyzed with regard to (1) paraphrase (what else could the interview partner have said), (2) manifest meaning (what is the manifest intention of what was said), (3) latent meaning (what might be the latent meaning behind what was said), (4) social relationship (what could what was said tell us about the social role distribution within the organization), and (5) potential consequences for the next unit of meaning (after what we know now, which information could the next unit of meaning possibly contain). Table 1 provides an overview of these steps.

The interpretation of the latent meaning is of particular importance, because according to the principles of objective hermeneutics, what is left out is often the key to understanding the meaning of a text. For instance, reading the text multiple times with emphasis on different words can lead to different interpretations. Likewise, the use of conditional clauses, active or passive voice, leaving out the subject in a sentence, switching between slang, everyday language and standard language, halfsentences, repetitions, the use of specific terms (e. g. is something an incident, an accident, a malfunction, a breakdown, a disaster, or a catastrophe), long breaks, etc. are all relevant when analyzing the meaning behind what was said.

In a second step, we built topic-related blocks to "attend the dynamics of the organization as the overall context" (see Lueger et al. (2005) for a detailed description of the methodology). This method enabled us to uncover the latent-meaning structures underlying social practices and therefore gave us an indication about the logics underlying change during the process. Whereas the first step aims at de-contextualizing, the second step concentrates on the structural conditions the statements reproduce and the implications that can be drawn from them for the entire organization. Throughout the interpretation processes we have developed hypotheses that have been challenged and constantly reflected step by step against the text (Flick 2002). Following Lueger et al. (2005) approved way of analysis was one quality measure; the other one was to keep a detailed protocol of our steps of analysis (see Table 2 for a selected example). 
Table 1 Interpretation scheme for fine analysis (Lueger et al. 2005, 1163)

\begin{tabular}{|c|c|}
\hline Steps & Typical questions to be asked \\
\hline 1. Paraphrase & $\begin{array}{l}\text { What is the information in the unit according to common sense? } \\
\text { Which issue has been chosen? }\end{array}$ \\
\hline $\begin{array}{l}\text { 2. Intention of the inter- } \\
\text { viewee }\end{array}$ & $\begin{array}{l}\text { Taking the viewpoint of the interviewee: } \\
\text { What could s/he want to say without explicitly speaking it out? } \\
\text { What meaning could it have for her/him? } \\
\text { What could s/he thereby want to point out to the interviewer? } \\
\text { What are her/his interests? }\end{array}$ \\
\hline $\begin{array}{l}\text { 3. Extensive interpreta- } \\
\text { tion: Latent elements of } \\
\text { the unit analysed and ob- } \\
\text { jective consequences for } \\
\text { behavior (or the system) } \\
\text { Explication of general } \\
\text { structures }\end{array}$ & $\begin{array}{l}\text { What are the different meanings that unfold if the unit is read with varying } \\
\text { intonation? } \\
\text { Linguistic characteristics: } \\
\text { Meanings of generalizations (like: one, everybody, people, etc.) } \\
\text { Are the verbs explicit enough (regarding who, whom, what)? } \\
\text { Specific grammar used: active/passive voice, conditional clauses, etc. } \\
\text { Other linguistic specificities: use of words, dialect, repetitions, breaking } \\
\text { off, slips of the tongue, etc. } \\
\text { Which people and issues are mentioned? } \\
\text { All other possible meanings of the unit } \\
\text { What could this statement mean in different social contexts? } \\
\text { What are the characteristics of a social system in which a statement like this } \\
\text { is meaningful? } \\
\text { Under which social conditions does a statement like this make sense? }\end{array}$ \\
\hline 4. Role distribution & $\begin{array}{l}\text { What actors (individuals, groups, organizations, etc.) are referred to (di- } \\
\text { rectly or indirectly) in the statement? } \\
\text { Which are their characteristics? } \\
\text { What is the relationship between them? } \\
\text { What are the consequences for the structure and dynamics of the system? }\end{array}$ \\
\hline $\begin{array}{l}\text { 5. What are the conse- } \\
\text { quences for the subse- } \\
\text { quent unit of meaning? }\end{array}$ & $\begin{array}{l}\text { What are the possibilities to carry on? How could the narrator proceed? } \\
\text { What statements could be expected? } \\
\text { Are there any restrictions? }\end{array}$ \\
\hline
\end{tabular}

Having identified a clear gap between how organization members say they perceive failure (failure is good ...) and how they actually perceive failure and deal with it (... as long as it does not happen), we continued our analysis on a broader level. We conducted further interviews and analyzed them in the context of the project and against the background of our initial hypotheses. Thus, in a second analytical round, all interview data, collected documents and observation notes were analyzed according to the rules of qualitative content analysis (Miles and Huberman 1994) with the aim of reconstructing typical organizational patterns of collective action, communication and decision-making. Additionally, the constant comparison with theory (Eisenhardt and Graebner 2007) served as a means to ensure validity and reliability according to case study research standards (Yin 2009). Consistent with standards of qualitative research approaches (Eisenhadt 1989), the research team spent considerable time as a group sharing impressions and data in order to achieve a consensual view of the results. 
Table 2 Selected example of unit of meaning analysis

\begin{tabular}{|c|c|c|}
\hline \multicolumn{3}{|c|}{ It was insufficiently considered by us (Es wurde von uns zu wenig bedacht) } \\
\hline 1 & Paraphrase & $\begin{array}{l}\text { We thought about that too little } \\
\text { We did not reflect enough } \\
\text { We did not fully anticipate } \\
\text { We gave too little consideration } \\
\text { It wasn't well-thought-out }\end{array}$ \\
\hline 2 & Intention & We failed to notice that \\
\hline 3 & Latent elements & $\begin{array}{l}\text { Passive voice ... nobody can be made responsible } \\
\text { We failed to notice, but maybe others did notice } \\
\text { We focused on something else } \\
\text { We underestimated the consequences/the importance } \\
\text { It seemed to be so easy } \\
\text { We did not consider what it takes from our side to implement the project after } \\
\text { the consultants leave } \\
\text { The consultants thought for us; we were not meant to think in the project; now } \\
\text { we have to think and act autonomously and now we don't know what to do } \\
\text { It is not reversible as we did not think about that at the beginning }\end{array}$ \\
\hline 4 & Role distribution & $\begin{array}{l}\text { By us } \rightarrow \text { who? The collective leadership board? The hospital? we and the } \\
\text { consultants? } \\
\text { The speaker did not decide alone, but sees his responsibility } \\
\text { Others are responsible too } \\
\text { By whom was it sufficiently considered? } \\
\text { Who could have warned them? }\end{array}$ \\
\hline 5 & $\begin{array}{l}\text { Subsequent } \\
\text { meaning }\end{array}$ & What has not been considered \\
\hline \multicolumn{3}{|c|}{ That it simply takes too much time, (dass da einfach zu viel Zeit draufgeht,) } \\
\hline 1 & Paraphrase & $\begin{array}{l}\text { Too much time is wasted } \\
\text { It takes too much effort } \\
\text { Nobody can return the time we have wasted } \\
\text { It swallows too much time }\end{array}$ \\
\hline 2 & Intention & Time is a critical resource and we do not want to waste it for that \\
\hline 3 & Latent elements & $\begin{array}{l}\text { Regardless of positive effects, it takes too much time } \\
\text { We do not have the time that is needed } \\
\text { Potential benefits of the project are not valued } \\
\text { We can't do it under these circumstances } \\
\text { It is useless to spend so much time on that }\end{array}$ \\
\hline 4 & Role distribution & What exactly takes too much time? \\
\hline 5 & $\begin{array}{l}\text { Subsequent } \\
\text { meaning }\end{array}$ & What is it that takes too much time? \\
\hline
\end{tabular}

\section{Results}

Our findings reveal how artifacts introduced as tools for schema change actually led to a decoupling of interpretive schemata and collective action and hence ultimately impeded change. In particular, we show how three subprojects aimed at improving how errors are dealt with were put into practice: the launch of an IT-based error reporting system, the employment of checklists, and the implementation of human factor training. We describe how these artifacts led to visible change on the surface and therefore legitimated that nothing changed; neither on the level of collective action nor for the enacted schema. The design of these artifacts satisfied the espoused 
Table 2 Selected example of unit of meaning analysis (Continued)

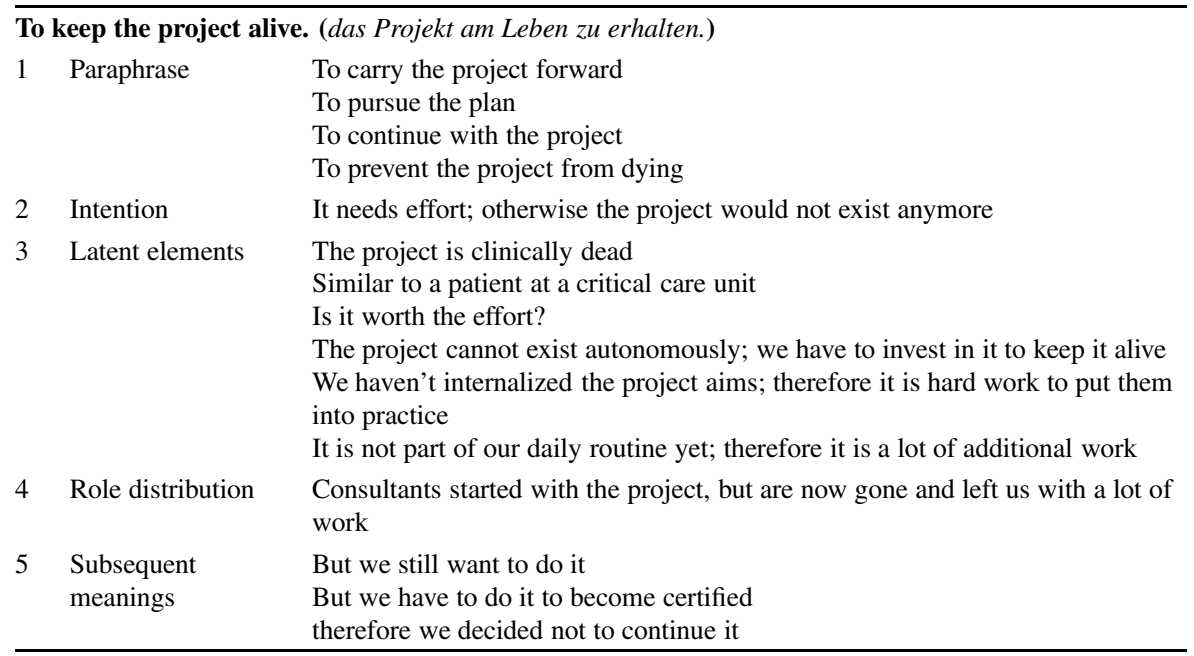

schema, while in their implementation they simultaneously strengthened the old, still enacted, schema. Hence, artifacts and their use allowed espoused and enacted schemata to coexist and to drift apart, which stabilized the status quo.

\subsection{Espousing a new Schema}

Espousing a new interpretive schema by articulating goals and expectations is a first step toward leading change. As noted earlier, espoused interpretive schemata are meant to provide meaning for everyday action and to guide how organizational members think and act. In our case, the espoused schema - primarily articulated by Riscure and the hospital's collective leadership board members - emphasized the vital function of learning from failure. In an internal document Riscure states: "Failure is good! Mistakes happen to all of us - all the time. We will help you to systematically learn from failure to improve quality" (internal document, Riscure: 1). The medical director of the hospital, responsible for implementing the project, emphasized what should be different after the project in the following way:

It (the new way of thinking - ann. the authors) aims at increasing awareness of a risk culture, for every employee of the hospital. It will lead to a better understanding of the necessity of constructive communication with real content between hierarchical levels and professional groups. (...) It raises awareness that making mistakes is a part of human nature. Everybody can make mistakes and everybody makes mistakes. (...) Mistakes should not be about blaming others and exposing them. That is not good. It's better to say, ok, this was a mistake and what can we do to prevent it from occurring again (medical director: 302). 
Table 3 Espoused versus enacted schema with regard to failure

\begin{tabular}{ll}
\hline Espoused schema & Enacted schema \\
\hline How we (say we) perceive failure & How we (actually) perceive failure \\
Failure is good & $\ldots$ as long as it doe not happen \\
Everybody is allowed to make mistakes & $\ldots$ but we will punish you for it \\
We have to learn from failure & $\ldots$ but we don't care if you hide it \\
\hline
\end{tabular}

The head of nursing used similar terms to describe the new espoused schema. In particular, she stated that:

We had the idea of establishing risk management in our hospital, which is primarily about avoiding mistakes and not about looking for them and blaming others. That was our first thought: creating a fundamentally new approach to not denounce people in a certain way, but to try to recognize a mistake before it happens and then simply correct or avoid it (head of nursing: 6).

Thus, decision makers of the consulting company, and the hospital clearly stated the project's goals and expectations. The new espoused schema was meant to convey a radically new attitude towards reporting errors and dealing with failure. The aim was to embrace failure as a source of learning by emphasizing that failure is good, that people are allowed to make mistakes, and that they should learn from failure. With regard to achieving that change, basic assumptions about communication across hierarchies and teamwork across professional groups needed to be addressed. The espoused schema also unveiled the shared core assumptions of the enacted schema, which closely associated failure with blame. It also emphasized that there seemed to be only limited content in the communication between hierarchical levels and fields of expertise. Thus the enacted schema was mainly based on hierarchical thinking and on isolated fields of expert status.

Although the implementation of new artifacts aimed at addressing these underlying issues, our results revealed that even after implementation there was still a gap between the espoused and the enacted schema regarding failure (see Table 3). On the basis of these initial results we analyzed how the artifacts had been implemented and thereby theorized about the interplay between artifacts, their use, interpretive schemata, and collective action.

\subsection{Artifacts and their use}

Artifacts play an important role in connecting "ways of thinking" and "collective action". To implement schema change Riscure relies on standardized tools. Their aim is to influence ways of thinking as well as collective action by introducing new artifacts: An IT-based error reporting system should open errors to discussion without blaming anybody in order to learn from failure. Creating and using checklists mainly aim at affecting collective action directly by improving interface communication between different fields of expertise and sub-groups. The human factor training is specifically designed to address the values and norms that guide the way the organization deals with failure. However, our findings revealed that although 
all these artifacts aimed at satisfying the newly espoused schema and thereby were intended to change the enacted schema they were created and used in a way that strengthened the enacted schema instead.

Implementing an IT-Based Error Reporting System FARES is an IT tool through which anybody can anonymously report errors or near-misses. Its purpose is to serve as a platform where errors and near-misses can be revealed "without risk" in order to use them as learning opportunities. Raising awareness of the importance of reporting an error or particularly a near-miss after it has happened, however, proved to be difficult. All our interview partners from hospital management overemphasized the fact that the error reporting system can be used anonymously. The quality manager of the hospital described FARES as a system where "they (medical staff and nursing staff - ann. the authors) can report any errors or near near-misses anonymously. (...) Access is anonymous. It is absolutely untraceable who reported what. You can add your name but you don't have to. You can do it anonymously" (106). Similarly, the head of nursing staff underlined, "it is vitally important that you communicate beforehand that everything is anonymous” (152). Anonymity should enhance the rate of reported errors, because nobody has to fear negative consequences. Riscure evaluates the errors and near-misses and sends the statistics to the hospital's board twice a year (medical director: 4). Based on international benchmarking data, Riscure provides recommendations for how to deal with the errors (webpage Riscure).

Although the espoused schema emphasized the importance of learning through failure, not even assuring absolute anonymity could persuade doctors and nursing staff to use FARES, the reporting system. Instead, anonymity allowed them to not use the system. One reason for the non-use is that reporting errors contradicts the still prevalent basic assumptions of the enacted schema, which emphasized that failure is a taboo and that near-misses are as much a taboo as actual errors. If errors or near-misses happen the social norm seems to be covering up for each other. An assistant medical director from the ophthalmological department (194) stated: "Show me the nurse that reports that a doctor has made a mistake. It will not happen, unless the doctor has some enemies". Another reason for the non-use is that the consequences of the report remain unclear; at least they are not communicated. Responding to our question as to what happens with the results of the reports one assistant medical director for surgery (291) replied: "Obviously there are already numbers. But I don't know them. There are parameters for measurement, but I don't know them. The risk assessors have them. I can't give you any feedback, numbers, or statistics". As a consequence, despite the assurance of anonymity there is almost no acceptance of the FARES system among the organization members. One nurse reported "actually I don't know anyone who uses it" (nurse gynecology: 156). Even the medical director (152) admitted "the acceptance could be better. I think we will have to reconsider if the system is good enough". Ironically he continued that he believes that "the employees would report more, if they were a hundred percent sure that their anonymity is warranted and that what they report leads to critical and fruitful discussions". Thus, people's fear of failure still remains and cannot be overcome by communicating that anonymity is guaranteed. 
Implementing Checklists The aim of introducing detailed checklists is to standardize behavior throughout the process of a specific treatment routine (e. g. a surgery) in order to avoid errors. Checklists serve as central tool in risk management projects and are even recommended by the WHO in order to minimize the risk of failure. Checklists define a "common wording" (Riscure webpage) and they also serve as a bridge between different hierarchical levels and professional groups with the aim of avoiding misunderstandings between these different areas of hierarchy and expertise. The head of nursing stated as one of the central aims of the implementation of checklists that everybody in the hospital should develop the same ideas about how to handle specific problems. Although some interview partners reported that the introduction of checklists has led to minor improvements, the head of nursing staff admitted that there were some problems with the project that were more massive than expected.

It was insufficiently considered by us, that it simply takes too much time to keep the project alive. We had massive difficulties with the checklists that were to become effective across the disciplines. The problem was to reduce them to a common denominator within a short time (head of nursing: 237).

Some units even started to set up their own checklists right before the project was implemented. "We knew in advance what would happen, and we didn't want to wait for the specialists to come and tell us what to do, so we created our own checklists" (assistant medical director, anesthesia: 40). Additionally, in some areas the impression prevailed that checklists were only for inexperienced "newbies". As one member of the nursing staff (47) put it: "Everything that's on the list is clear to us. Except maybe to new employees. But when you have been around for a longer time, everything has become routine". Hence, standardizing individual behavior and communication across hierarchical and functional borders was difficult: "Too many cooks spoil the broth. Take for example a checklist where the internist, the surgeon, the anesthesiologist, the radiologist, and a couple of nursing staff are participating and they need to come to an agreement about what goes on the checklist. That was a really hard way for us and sometimes we failed" (head of nursing: 230). As a consequence, multiple checklists for separate areas were set up. These multiple checklists hampered the aim of achieving standardization and facilitated the parallel existence of multiple views on what matters during the collective execution of a routine. "We couldn't implement particular checklists in the way we wanted, but in a slimmed down version or in two different versions for two areas, when it was absolutely impossible for those areas to reach an agreement" (head of nursing: 238).

Setting up multiple checklists for different areas of expertise actually prevents communication and sustains hierarchical communication barriers. At the same time, multiple checklists secure the autonomy and expertise of medical staff. Medical staff and nursing staff still do not cooperate and use the same wording during treatment routines but rather act in parallel. "It's about communication. The hierarchy tells us that it has to be the doctor who says what is to be done. The nurses look at him/her and wait for orders. A nurse does not dare to ask multiple times, even when she didn't fully understand and then maybe she makes a mistake" (quality manager: 254). Consequently, although the espoused schema emphasizes openness for communication 
across hierarchical and professional levels, the adaptation of checklists for different professional groups contributes to sustaining established hierarchical thinking and the protection of expertise.

Human Factor Training Human factor training aims at creating awareness that making mistakes is human. Mistakes are not related to professional incompetence but to communication deficits, poor teamwork or insufficient decision-making processes. Therefore, one goal of training is to improve the collaboration between medical staff, nursing staff, and administrative staff and to reduce barriers that are created through the hierarchical structure at the hospital by creating a common understanding of terms in use: "It is important that the term 'acute' means the same for everybody. Acute is acute and it does not just last one minute or twenty minutes, but it has to mean the same thing to everybody" (head of nursing: 51).

All interview partners reported positive feelings vis-à-vis the human factor training. They perceived it as interesting and fun: "People said, 'let's be surprised. It's going to be fun.' The human factor training has been regarded as a nice change of pace, but it did not have actual implications" (nurse gynecology: 192). Human factor training achieved the goal of raising the awareness of potential risks and underlined double-checking whether a sample of blood really matches the person at hand and the necessity of paying more attention to actions that are perceived as routine and are therefore a source of errors due to carelessness. However, the transfer to daily routines that involve different subgroups was hampered by the fact that this issue was not covered by the training structure:

It was called human factor training and it was actually a lecture and not training. To me training means that you learn something or ... that something is taught practically, but that was actually a lecture by these two men that took place under this name (nurse gynecology: 192).

Human factor training supported the change project on the surface but failed to change the basic way of communicating due to the way the training was implemented. The consultants aimed at transferring standards from the aviation industry to the hospital, which - aside from similarities such as a "culture of expertise" - is simply a very different context. While in aviation checklists are applied to standardized processes of flying an aircraft, surgical procedures on humans in hospitals are not as easy to standardize. Some interview partners emphasized what they perceived as the limits of standardization in a health care context. "Standardization can also be overdone. Every medical emergency is different. Always. There is a danger of going too far in trying to spell out every single step" (assistant doctor, gynecology: 97).

\subsection{Enacting the old Schema}

Our findings indicate that all three artifacts and the way they were introduced missed the target of changing the organization members' attitudes towards failure. Failure remains an organizational taboo. The social norms of dealing with failure are still that errors must not happen at all and if they happen they have to be covered up. 
We found different reactions vis-à-vis artifacts that strive to influence interpretive schemata and collective action. The use of FARES attempts to influence the interpretive schema in terms of viewing errors as good, and collective action by reporting them. However, since there is still a fear of failure in any form, errors are not reported. Anonymity is important to remove the fear of failure; however, emphasizing anonymity also implies that talking about failure is still not allowed. At the same time anonymity allows for not using the system - users do not even know if someone has used it as reports about or the consequences of registered errors are not communicated. Thus, the way of implementing such an artifact that aims at addressing interpretive schema change ultimately leads to a stabilization of the old enacted schema, whilst satisfying the espoused schema.

Implementing checklists has a direct impact on how the treatment routine is performed and therefore provoked various activities that led staff members to embrace checklists on a superficial level. In fact, the checklists actually hamper communication and sustain hierarchical communication barriers and therefore facilitate the perpetuation of the established enacted schema.

The aim of the human factor training, finally, was to address the interpretive schema with regard to how to perceive and deal with errors. The way human factor training was conducted met the goal of raising awareness, but was not sufficient to alter the basic ways of working together in the daily routine.

Overall, the change project built on the idea of transferring safety standards from the aviation industry to the health care context by espousing a new schema that aims at incorporating new values and expectations. Regarding the approach towards the project the medical director stated that "the consultants were inspecting the company and they talked with department heads and teams about possible problems. There are risks that can become manifest, and damage may occur from the risk. Based on that, expectations were formulated, so we said what we want to implement" (medical director: 60-62). He added that regarding the implementation of the project "the intellectual approach was not to do a top-down method" (medical director: 45).

The rationale behind this approach was that the new values of participation and a better cross-hierarchical communication flow should already characterize the implementation of the change project and thereby reduce anxiety and stimulate openness for change. However, the way change was actually introduced into the organization reflected the overall hospital atmosphere that is founded on formal authority, hierarchical thinking and expertise (see Table 4).

Although the medical director emphasized the espoused value of participation, organization members stressed that management, including the consultants, simply presented them with faits accomplis. One assistant doctor (75) claimed "because as an assistant doctor I am the lowest social class in the hospital, I was not involved at all." An assistant medical director (ophthalmology: 5) illustrated how participation was framed "We had an event, where everyone in the hospital was obliged to participate, and where the firm Riscure was presented." Stimulating open communication was a central aim as well and an espoused expectation of the new project. "It's about communication. The hierarchy tells us that it has to be the doctor who says what is to be done. The nurses look at him/her and wait for orders. A nurse does not 
Table 4 Espoused versus enacted schema with regard to how change is implemented

\begin{tabular}{ll}
\hline Espoused schema & Enacted schema \\
\hline Participation: We want everybody to participate & $\begin{array}{l}\text {... but we already know what to do and we will tell } \\
\text { you via hierarchical order }\end{array}$ \\
$\begin{array}{l}\text { Communication: We want to stimulate open com- } \\
\text { munication across hierarchical levels and fields of } \\
\text { expertise }\end{array}$ & $\begin{array}{l}\text {... but it is ok if you take different measures for } \\
\text { different hierarchical levels and fields of expertise }\end{array}$ \\
$\begin{array}{l}\text { Openness: We want to change } \\
\text {... but there are no sanctions if you act like you } \\
\text { have always done }\end{array}$ \\
\hline
\end{tabular}

dare to ask multiple times, even when she didn't fully understand and then maybe she makes a mistake" (quality manager: 254).

Yet the doctors continue to enact their old schemata "We are lone fighters. (...) There are no mutual controls" (assistant medical director, anesthesia: 311). Hence, although the new espoused schema emphasizes openness, the new artifacts are particularly open for interpretation. The way they are implemented and used inhibits change. The gap between espoused and enacted schema is still present in the statements that were made after the completion of the project:

As I said before, it (the project - ann. the authors) strengthened the awareness of the need to concentrate on not mistaking patients, not confusing test laboratory samples. It made us think about sources of error and where to be extremely careful. However, once it had finished, the flaws of daily routine returned (nurse gynecology: 218).

\subsection{Artifacts and their Role in Decoupling Espoused and Enacted Schema}

To sum up, the espoused schema officially expresses a new way of dealing with failure and formulates new social expectations. This newly espoused schema, however, is almost diametrically opposed to the old, still enacted, schema. New artifacts, such as an error reporting system, checklists, and training sessions, have been implemented to formally support the espoused schema, but were designed in a way that actually facilitates enacting the old schema. In particular, the newly generated artifacts enable actors to sustain their execution of organizational routines (see Fig. 1). As a consequence, artifacts and their use decouple espoused and enacted schemata instead of connecting them. The decoupling of espoused and enacted schemata leads to a state of anti-learning and serves as a means to maintain the status quo whilst pretending change.

\section{Discussion}

Avoiding errors or at least learning from failure in health care organizations is of vital importance for patient safety. Establishing a culture that facilitates learning, however, requires health care organization members to adopt new ways of thinking and to change collective action. The idea that organizational interpretive schemata and collective action of organization members are tightly interrelated is increasingly 


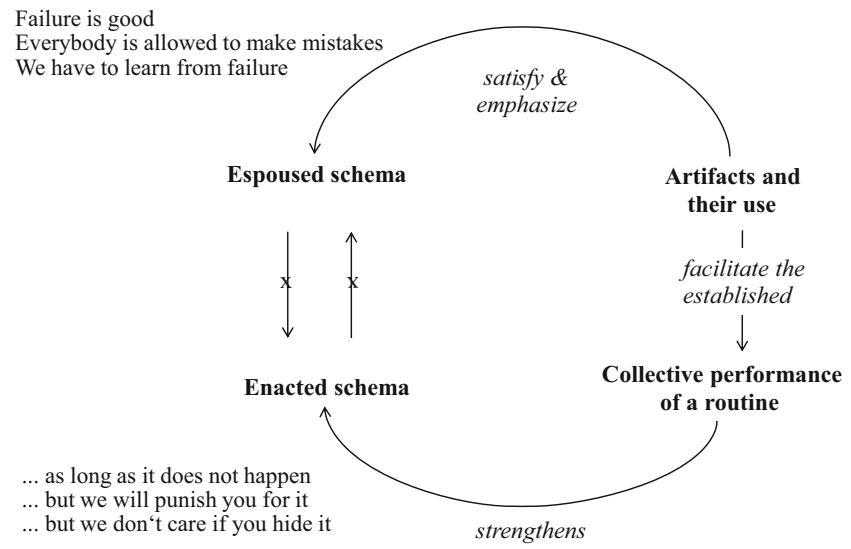

Fig. 1 Artifacts as linking pins between interpretive schemata and collective action

attracting the attention of researchers. While Rerup and Feldman (2011) show how espoused and enacted schemata converge through trial-and-error processes, this study proposes that the creation of artifacts may serve as a means to interrupt this interrelatedness. Although on the surface everyone would appreciate changes in treatment routines that help to avoid errors, the guiding norms of individual vigilance and self-centeredness and a culture that emphasizes hierarchy as a core value, as well as the lack of sanctions for enacting the old schema, led to a situation, where the new espoused schema was never enacted. Our empirical study thus highlights the role of artifacts and how their use can decouple espoused and enacted schema.

Interpretive Schemata and Organizational Change Our findings elucidate barriers behind interpretive schema change. Current research is well aware of the difficulties of changing interpretive schemata (e. g. Reger and Palmer 1996; Weber and Crocker 1983). However, focusing on how divergence between espoused and enacted schemata is created and sustained can serve as a new approach for explaining failure in attempts to adapt interpretive schemata. Previous work emphasizes that changing interpretive schemata can work in different ways (see e. g. Labianca et al. 2000, 251). The "synthesis approach" assumes that schema change is the result of a dialectic process, in which a conflict between two groups (one that champions the old schema and one that champions the new one) is resolved by developing a new schema that is a synthesis of both (Bartunek 1984). The "replacement approach", in contrast, assumes that a schema change is a more radical process, in which a new schema replaces an old one (Labianca et al. 2000). In both approaches, schema change works because there is a recursive relationship between interpretive schemata and collective action patterns.

We show that the introduction of new artifacts in a way that facilitates decoupling the interpretive schema from collective action leads to interruption of this recursive relationship, which causes change projects to fail. This is possible because the artifacts are designed in a way to fit the organizational culture and to preserve hierarchical barriers. 
Identity, Culture, and Organizational Change Our findings also contribute to the discourse on identity and change. Previous research found out that organizational identity serves as a powerful barrier to change (Dutton and Dukerich 1991; Gioia and Thomas 1996; Nag, Corley, and Gioia 2007; Reger, Gustafson, Demarie, and Mullane 1994). We can add to this literature stream by emphasizing that this effect is particularly strong whenever change affects not only the organizational identity, but also and especially the professional identity and self-concept of the actors concerned. Tucker and Edmondson (2003) showed that the health care industry has developed the social norm of individual vigilance, which encourages independence but also prevents medical as well as nursing staff from discussing errors or near-misses openly. They (Tucker and Edmondson 2003, 61) particularly emphasize that, as a result of the hierarchical structure, one core rule of thumb among nurses was "to ask for help from people who were socially close rather than from those who were best equipped to correct the problem". We connect to this finding by showing that the social norms of individual vigilance and hierarchical separation in communication are shared across hierarchical layers and that they are so deeply embedded in the self-concept of nursing staff and medical staff that these prevalent norms serve as powerful barriers against change attempts.

Additionally, our findings connect to the concept of organizational routines' embeddedness. Howard-Grenville $(2005,619)$ uses the notion of embeddedness, which describes the degree to which a routine overlaps with the enactment of other organizational structures, to analyze the likelihood of change in a routine over time. Our findings indicate that the embeddedness of routines in a cultural frame that emphasizes hierarchy as a core value promotes stickiness and prevents these routines from changing. Individual orientations towards a routine, as partly manifested in our case by medical and nursing staff, might differ slightly, and therefore induce at least some amount of variation in the routine performance. Over time, however, in hierarchical settings the more powerful agents will exploit or even create ambiguity to enforce their interpretations and attempts to change collective actions will suffer a backlash.

Artifacts, Sanctions, and Organizational Change We also contribute to work on artifacts in performing collective organizational action (D'Adderio 2008, 2011; Rafaeli and Vilnai-Yavetz 2004; Turner and Rindova 2012). In their examination of "the folly of designing artifacts, while hoping for patterns of action", Pentland and Feldman $(2008,235)$ already highlighted that the assumption that designing artifacts produces repetitive patterns of collective action represents "technological determinism", which contradicts the complexity of collective action patterns. We extend this view by showing how creating artifacts may even serve to disconnect interpretive schemata from collective action.

Hence, ambiguity in designing and implementing artifacts may support resistance to change in a well-concealed manner. Such artifacts may then be designed or interpreted to support existing social expectations and action patterns. Thus, our findings contribute to research that focuses on the role artifacts play in implementing change. In contrast to studies that focus on how artifacts enable responsiveness to change (e. g. Turner and Rindova 2012), we emphasize their function as legitimacy- 
preserving symbols that can become decoupled from the collective action patterns that constitute an organizational routine. This process of decoupling facilitates parallelism of competing individual orientations (Gilbert 2006). With their enactment, powerful individuals provide their own guide of how artifacts are to be interpreted.

Finally, our findings indicate that artifacts which are meant to change collective action need to be accompanied by sanctions to unfold their impact. Thus, our study contributes explanations as to why leading change by implementing new formal rules (e. g. the mandatory use of checklists during surgery) does not always produce the desired effects. Although current conceptualizations of organizational routines (cf. e. g. Feldman 2003), draw heavily on insights from structuration theory, where sanctions play an important role, previous studies on the impact of artifacts on behavior have remained relatively silent in explaining rule-following and rule-deviating behavior. Without mechanisms that protect formal rules against violation, introducing new formal rules may be insufficient to drive change, especially if the new rules contradict existing norms and the self-concept of organization members (Geiger and Schröder 2014).

\section{Limitations, Practical Implications, and Outlook}

Our study connects interpretive schemata, i. e. shared assumptions, artifacts and collective action. We do so by combining the methodology of objective hermeneutics with more established methods of qualitative data analysis. We are aware that, despite having a long tradition in sociological research, objective hermeneutics is a relatively new approach for theory building in organization science. However, we think that this approach can be regarded as a further step towards method triangulation in examining qualitative data. We see it as useful interpretative method for complementing prevalent positivistic approaches (Petit and Huault 2008).

Objective hermeneutics is one option to satisfy the call to identify generalizable patterns of behavior (cf. Lueger et al. 2005) on the one hand and to offer deep insights into single case studies on the other. It facilitates making the deep structure of a text subject to discussion, which is particularly useful when the research focus lies on concepts such as shared assumption, social expectations, organizational values, or power relationships (Lueger et al. 2005). Such concepts are deeply embedded in the organizational culture and often are not discussed openly; sometimes interview partners are not even conscious of them. Approaches that could provide better insights into such phenomena should be appreciated.

Additionally, one could argue that generalizations from single case study findings are always somewhat problematic. We tried to overcome this potential limitation by providing "thick descriptions", by deducing some simple principles with universal applicability in similar situations, and with extensive comparison of our findings with literature. The latter tactic can be regarded as a mild form of cross case analysis (Yin 2009) that further enhances the robustness of the findings.

Our findings have some implications for practice. Attempts to guide the change of collective action by implementing new artifacts might in some cases be a folly (Pentland and Feldman 2008); yet they are common practice (cf. Güttel et al. 2012; 
Friesl and Larty 2013). Most kinds of change projects - more or less successfully - focus on this approach. Our findings provide a further lens for analyzing failed change projects. When powerful actors, i. e. those who create artifacts, observe their implementation, and sanction compliance with them, (ab)use their power for decoupling schemata and action, change projects are doomed to fail. A coherent policy when implementing new formal rules accompanied by clear sanctions and the willingness to punish deviations can align employee behavior if cultural values and norms do not undermine this endeavor.

However, if resistance, e. g. not reporting errors, originates from anxieties which are deeply embedded in the organizational culture change efforts need to address causes on the cultural level before changing artifacts on the surface of an organization. Changing the organizational culture before implementing new artifacts will consequently serve as a pathway to changing behavior that avoids a decoupling of interpretive schemata and collective action. This finding helps to explain difficulties that firms have when trying to implement change, but it is also particularly applicable to change projects in the public administration sector. The logic of decoupling inhibits change in small projects as well as in large economically relevant projects, such as the reform of the health care services or the education system.

\section{Conclusion}

Until now, interpretive schemata and organizational routines have been largely treated as two separate research streams. Current research increasingly emphasizes their tight interconnectedness (Rerup and Feldman 2011, 606; Howard-Grenville 2005; Tucker and Edmondson 2003). Our paper provides a further step towards the integration of both research streams. While Rerup and Feldman (2011) show how espoused and enacted schemata converge through trial-and-error processes, our findings suggest that the creation of artifacts that officially are aimed at changing interpretive schemata may serve as a means to interrupt this interrelatedness. We have explained how artifacts are used to stabilize a divergence between espoused and enacted schemata. By focusing on the process of decoupling schemata from action we have addressed mechanisms that may cause change projects of all sizes to fail and provided an analytical lens for investigating the logic of failure.

Open Access This article is distributed under the terms of the Creative Commons Attribution 4.0 International License (http://creativecommons.org/licenses/by/4.0/), which permits unrestricted use, distribution, and reproduction in any medium, provided you give appropriate credit to the original author(s) and the source, provide a link to the Creative Commons license, and indicate if changes were made.

\section{References}

Balogun, Julia, and Gerry Johnson. 2005. From intended strategies to unintended outcomes: The impact of change recipient sensemaking. Organization Studies 26:1573-1601.

Bartunek, Jean M. 1984. Changing interpretive schemes and organizational restructuring: The example of a religious order. Administrative Science Quarterly 29:355-372.

Bartunek, Jean M., Catherine A. Lacey, and Diane R. Wood. 1992. Social cognition in organizational change: An insider/outsider approach. Journal of Applied Behavioral Science 28:204-223. 
Becker, Markus C. 2004. Organizational routines: A review of the literature. Industrial \& Corporate Change 13:643-677.

D'Adderio, Luciana. 2008. The performativity of routines: Theorising the influence of artifacts and distributed agencies on routines dynamics. Research Policy 37:769-789.

D'Adderio, Luciana. 2011. Artifacts at the centre of routines: Performing the material turn in routines theory. Journal of Institutional Economics 7:197-230.

Dutton, Jane E., and Janet M. Dukerich. 1991. Keeping an eye on the mirror: Image and identity in organizational adaptation. Academy of Management Journal 34:517-554.

Eisenhardt, Kathleen M. 1989. Building theories from case study research. Academy of Management Review 14:532-550.

Eisenhardt, Kathleen M., and Melissa E. Graebner. 2007. Theory building from cases: Opportunities and challenges. Academy of Management Journal 50:25-32.

Elsbach, Kimberly D., Pamela S. Barr, and Andrew B. Hargadon. 2005. Identifying situated cognition in organizations. Organization Science 16:422-433.

Feldman, Martha S. 2003. A performative perspective on stability and change in organizational routines. Industrial and Corporate Change 12:727-752.

Feldman, Martha S. 2004. Resources in emerging structures and processes of change. Organization Science 15:295-309.

Feldman, Martha S., and Brian T. Pentland. 2003. Reconceptualizing Organizational Routines as a Source of Flexibility and Change. Administrative Science Quarterly 48:94-118.

Flick, Uwe. 2002. Qualitative research - state of the art. Social Science Information 41:5-24.

Friesl, Martin, and Joanne Larty. 2013. Replication of routines in organizations, existing literature and new perspectives. International Journal of Management Reviews 15:106-122.

Gawande, Atul. 2011. The checklist manifesto: How to get things right. New York: MacMillan.

Geiger, Daniel, and Anja Schröder. 2014. Ever-changing routines? Toward a revised understanding of organizational routines between rule-following and rule-breaking. Schmalenbach Business Review 66:170-190.

Gilbert, Clark G. 2006. Change in the presence of residual fit: Can competing frames coexist? Organization Science 17:150-167.

Gioia, Dennis A., and James B. Thomas. 1996. Identity, image, and issue interpretation: Sensemaking during strategic change in academia. Administrative Science Quarterly 41:370-403.

Gioia, Dennis A., James B. Thomas, Shawn M. Clark, and Kumar Chittipeddi. 1994. Symbolism and strategic change in academia: The dynamics of sensemaking and influence. Organization Science 5:363-383.

Güttel, Wolfgang H., Stefan W. Konlechner, Barbara Müller, Julia K. Trede, and Mark Lehrer. 2012. Facilitating ambidexterity in replicator organizations: Artifacts in their role as routine-recreators. Schmalenbach Business Review 74:187-203.

Hildebrand, Bruno. 2004. Gemeinsames Ziel, verschiedene Wege: Grounded Theory und Objektive Hermeneutik im Vergleich. Sozialer Sinn 4:177-194.

Hitzler, Ronald. 2005. The reconstruction of meaning: Notes on German interpretive sociology. Forum: Qualitative Social Research 6:1-10.

Howard-Grenville, Jennifer A. 2005. The persistence of flexible organizational routines: The role of agency and organizational context. Organization Science 16:618-636.

Isabella, Lynn A. 1990. Evolving interpretations as a change unfolds: How managers construe key organizational events. Academy of Management Journal 33:7-41.

Labianca, Giuseppe, Barbara Gray, and Daniel J. Brass. 2000. A grounded model of organizational schema change during empowerment. Organization Science 11:235-257.

Leidner, Robin. 1993. Fast food, fast talk: Service work and the routinization of everyday life. Berkeley: University of California Press.

Lewis, Geraint A., Rhema Vaithianathan, Peter M. Hockey, Guy Hirst, and James P. Bagian. 2011. Counterheroism, common knowledge, and ergonomics: Concepts from aviation that could improve patient safety. The Milbank Quarterly 89:4-38.

Lueger, Manfred, Karl Sandner, Renate Meyer, and Gerhard Hammerschmid. 2005. Contextualizing influence activities: An objective hermeneutical approach. Organization Studies 26:1145-1168.

Miles, Matthew B., and Michael A. Huberman. 1994. Qualitative data analysis: An expanded sourcebook, 2nd edn., Thousand Oaks: Sage.

Nag, Rajiv, Kevin G. Corley, and Dennis A. Gioia. 2007. The intersection of organizational identity, knowledge, and practice: Attempting strategic change via knowledge grafting. Academy of Management Journal 50:821-847. 
Nystrom, Paul C., and William H. Starbuck. 1984. To avoid organizational crises, unlearn. Organizational Dynamics 12:53-65.

Oevermann, Ulrich, Tilman Allert, Elisabeth Konau, and Jürgen Krambeck. 1979. Die Methodologie einer "objektiven Hermeneutik" und ihre allgemeine forschungslogische Bedeutung in den Sozialwissenschaften. In Interpretative Verfahren in den Sozial- und Textwissenschaften, ed. Hans-Georg Soeffner, 352-434. Stuttgart: Metzler.

Parmigiani, Anne, and Jennifer Howard-Grenville. 2011. Routines revisited: Exploring the capabilities and practice perspectives. Academy of Management Annals 5:413-453.

Pentland, Brian T., and Martha S. Feldman. 2008. Designing routines: On the folly of designing artifacts, while hoping for patterns of action. Information and Organization 18:235-250.

Petit, Sandra C., and Isabelle Huault. 2008. From practice-based knowledge to the practice of research: Revisiting constructivist research works on knowledge. Management Learning 39:73-91.

Rafaeli, Anat, and Iris Vilnai-Yavetz. 2004. Emotion as a connection of physical artifacts and organizations. Organization Science 15:671-686.

Reger, Rhonda K., Loren T. Gustafson, Samuel M. Demarie, and John V. Mullane. 1994. Reframing the organization: Why implementing total quality is easier said than done. Academy of Management Review 19:565-584.

Reger, Rhonda K., and Timothy B. Palmer. 1996. Managerial categorization of competitors: Using old maps to navigate new environments. Organization Science 7:22-39.

Rerup, Claus, and Martha S. Feldman. 2011. Routines as a source of change in organizational schemata: The role of trial-and-error learning. Academy of Management Journal 54:577-610.

Salvato, Carlo, and Claus Rerup. 2011. Beyond collective entities: Multi-level research on organizational routines and capabilities. Journal of Management 37:468-490.

Seidl, David, and Kai H. Becker. 2006. Organizations as distinction generating and processing systems: Niklas Luhmann's contribution to organization studies. Organization 13:9-35.

Siggelkow, Nicolaj. 2007. Persuasion with case studies. Academy of Management Journal 50:20-24.

Tamuz, Michael, and Eric J. Thomas. 2006. Classifying and interpreting threats to patient safety in hospitals: Insights from aviation. Journal of Organizational Behavior 27:919-940.

Tucker, Anita L., and Amy C. Edmondson. 2003. Why hospitals don't learn from failures: Organizational and psychological dynamics that inhibit system change. California Management Review 45:55-72.

Turner, Scott F., and Violina Rindova. 2012. A balancing act: How organizations pursue consistency in routine functioning in the face of ongoing change. Organization Science 23:24-46.

Wagner, Stephan M., Peter Lukassen, and Matthias Mahlendorf. 2010. Misused and missed use Grounded Theory and Objective Hermeneutics as methods for research in industrial marketing. Industrial Marketing Management 39:5-15.

Weber, Reneé, and Jennifer Crocker. 1983. Cognitive processes in the revision of stereotypic beliefs. Journal of Personality and Social Psychology 45:961-977.

Yin, Robert K. 2009. Case Study Research, Design and Methods, 4th edn., Thousand Oaks: Sage. 